hearing loss. Infected infants received one-year therapy (pyrimethamine/sulfadiazine); 1/13 infant developed neutropenia as adverse therapy effect.

At a median age of 2 years all infected infants had a normal psychomotor development (range $1-10$ years).

Conclusions It is advisable to perform IgM/IgG-WB on infant serum and the compared analysis for mother-infant pairs within the first month of life when high risk factors for Toxoplasmosis transmission are present.

\section{ANTIBIOTIC RESISTANCE AND PNEUMOCOCCAL CONJUGATED VACCINES COVERAGE OF STREPTOCOCCUS PNEUMONIA FROM MIDDLE EAR FLUID OF CHILDREN $<5$ YEARS}

doi:10.1136/archdischild-2012-302724.0925

1,20 Falup-Pecurariu, ${ }^{3}$ E Leibovitz, ${ }^{2} \mathrm{~A}$ Mercas, ${ }^{2} \mathrm{R}$ Lixandru, ${ }^{2} \mathrm{~L}$ Bleotu, ${ }^{2} \mathrm{C}$ Zavarache, ${ }^{4} \mathrm{C}$ Falup-Pecurariu, ${ }^{3} \mathrm{~N}$ Porat, ${ }^{3} \mathrm{R}$ Dagan, ${ }^{3} \mathrm{D}$ Greenberg. ${ }^{1}$ Department of Pediatrics, Faculty of Medicine, Transilvania University; ' 2 Department of Pediatrics, University Children's Hospital, Brasov, Romania; ${ }^{P}$ Pediatric Infectious Disease Unit, Soroka University Medical Center, Ben-Gurion University, Beer-Sheva, Israel; ${ }^{4}$ Faculty of Medicine, Transilvania University, Brasov, Romania

Aims A prospective study was initiated in Brasov, Romania in 2009 to assess the antibiotic resistance pattern of Streptococcus pneumoniae (Pnc) isolated from middle ear fluid in children with acute otitis media (AOM) < 5years old.

Methods Patients diagnosed with AOM who underwent tympanocentesis or presented with purulent otorrhea of $<24$ hours duration were enrolled.

Results 206 patients were enrolled, 132 (64\%) episodes occurred in children < 2years old; 105 (51\%) were culture-positive. 108 isolates were recovered: Pnc - 75 (67\%), H. influenzae - 26 (24\%) and others - 7 (9\%). Nonsusceptibility to penicillin was found in 25/27 (93\%) $[\mathrm{MIC}>1.5 \mu \mathrm{g} / \mathrm{mL}])$. Pnc resistance to TMP/SMX, erythromycin and clindamycin and MDR (multidrug resistance) were 22/27 (82\%), $16 / 27$ (59\%), 13/27 (48\%) and 15/27 (56\%), respectively. Of the 39 (54\%) Pnc serotyped the most common were: 19F (26\%), 6B (18\%), $14(15 \%), 23 \mathrm{~F}(15 \%)$ and 19A (8\%). Penicillin highly resistant was found in $84.6 \%$ (11/13): 2-6B, 6-19F, 2-14 all included in the PCV 7-valent (PCV7), except for 2 isolates: 9A, 22F. Out of the 13 highly resistant serotypes 7 (53.84\%) were multi drug resistant and all of them were $6 \mathrm{~B}$ or $19 \mathrm{~F}$. $35 / 39(90 \%)$ of all SP isolates are included in PCV-13.

Conclusions The proportion of penicillin resistance Pnc isolated from MEF was extremely high as well as resistance to other common antibiotics. Coverage of PCV7 and PCV10 vaccines was equal. The PCV13 coverage was $90 \%$. Most antibiotic resistant serotypes were included in the PCV13.

\section{HAEMOPHILUS INFLUENZAE IN CHILDREN: RESISTANCE TO SIX OTHER BÊTA-LACTAMS AMIONG AMPICILLIN-RESISTANT STRAINS}

doi:10.1136/archdischild-2012-302724.0926

S Mzilem, H Smaoui, A Kechrid. Children's Hospital of Tunis, Tunis, Tunisia

Background and Aims Haemophilus influenzae (Hi) is a human pathogen responsible for various infections in both children and adults.

We describe in this study the susceptibility patterns and $\beta$-lactam resistance mechanisms of 62 ampicillin-resistant H.i strains isolated from children at the children's hospital of Tunis during 2009 and 2010.

Materials and Methods All strains were identified and serotyped using conventional methods. Antimicrobial susceptibility was determined by E-test. The antibiotics tested were amoxicillin, amoxicillin-clavulanate, cefixim, cefuroxim, cefotaxim, cefpodoxim and imipenem. The $\beta$-lactamase production was performed using the nitrocefin test. We determined the resistance genes $\left(b l a_{\mathrm{TEM}-1}\right.$, bla $a_{\text {ROB-1 }}$ and $f t s$ I) by PCR.

Results Isolates were identified as non capsulated and were classified into 3 groups according to their $\beta$-lactam resistance mechanisms: $\beta$-lactamase positive ampicillin-resistant (BLPAR: $50 \%$ ); $\beta$-lactamase negative ampicillin-resistant (BLNAR: 40.32\%) and $\beta$-lactamase positive amoxicillin-clavulanate-resistant (BLPACR: 9.68\%). All strains showed high amoxicillin, amoxicillin-clavulanate, cefuroxim and imipenem MICs. Among these, the less active one was imipenem with $\mathrm{MIC}_{50}>32 \mathrm{mg} / \mathrm{l}$ in all strains. The highest MICs of cefuroxim were in BLPACR strains $(2-4 \mathrm{mg} / \mathrm{l})$. MICs ranges of this antibiotic were $0.5-6 \mathrm{mg} / \mathrm{l}$ in BLNAR and $0.125-4 \mathrm{mg} / \mathrm{l}$ in BLPAR. Cefotaxim, cefixim and cefpodoxim were the most active agents tested against our strains.

Conclusion This study indicates that many $\beta$-lactams are ineffective among some Hi strains. So, it's important to have an appropriate usage of antibiotics to stop these phenomena. We must make other investigations to know if these strains belonged to the same clone or if it's a question of an outbreak in our hospital.

\section{RHINOVIRUS INFECTIONS IN HIGH-RISK CHILDREN}

doi:10.1136/archdischild-2012-302724.0927

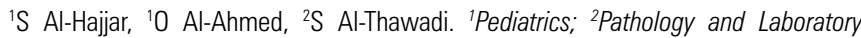
Medicine, King Faisal Specialist Hospital \& Research Centre, Riyadh, Saudi Arabia

Background Human rhinoviruses (HRVs) are recognized as major cause of cold and flu-like illness.

Objectives To analyze the clinical features and disease burden for children with underlying medical disorders and documented HRVs infections.

Methods This is a retrospective study that include 48 children who were hospitalized for acute respiratory illnesses in KFSHRC between October 2007 and June 2010. HRVs in nasopharyngeal aspirates, swabs or Bronchoalveolar lavage were detected by nucleic acid detection tests in addition to 13 common respiratory viruses.

Results HRV was the most frequently detected virus $27 \%$ $(48 / 181)$ in hospitalized children with acute respiratory disease. $63 \%$ of patients had chronic medical conditions and $37 \%$ of patients had immunocompromising conditions. The median age was 22 months, 58\% were male. HRV showed broad seasonal activity. The peak incidence was in November, December and June. The most common symptoms were cough (58\%), fever $(56 \%)$, dyspnea $40 \%$ and running nose $(25 \%)$. Crepitation and wheezes, were present in $23.9 \%, 20.8 \%$, respectively. Twentytwo of 48 patients (46\%) had chest radiographic abnormalities, most commonly atelectasis or lobar infiltrate. Seventeen $(35 \%)$ patients needed intensive care unit (ICU) admission and thirteen (76\%) required mechanical ventilation, there were two bacterial and one fungal co-infection documented in this patient. The mean duration of ICU stay was 17.9 days. Fifteen (88\%) of the HRV-positive patients survived, while 2 (12\%) patients died. Co-infection with other viral respiratory pathogens was common $(17 \%)$.

Conclusion HRVs were associated with severe lower respiratory tract infection and hospitalization in children with chronic or immunocompromising conditions.

\section{NEUTROPHIL CD64 INDEX (CD64IN) IN CEREBROSPINAL FLUID IN DIAGNOSING BACTERIAL VENTRICULITIS IN CHILDREN WITH EXTERNAL VENTRICULAR DRAINAGE}

doi:10.1136/archdischild-2012-302724.0928 